\title{
MENSCHLICHES LEBEN ZWISCHEN THERAPIE UND TECHNIK
}

Bernhard Waldenfels*

SÍNTESE - Certamente, desde que o ser humano existe, a vida nunca esteve isenta de manipulação técnica. Todavia, formas recentes de biotecnologia vão mais longe, tendendo a tornar indiscernível as distinções clássicas entre crescimento natural e produção artificial. Ars sive natura, este poderia ser o novo slogan. Neste sentido, em oposição à bioética corrente que tenta compensar o monismo tecnológico através de um dualismo prático, discutiremos a idéia de que, quando age sobre o Outro (Fremdeneinwirkung), a biotecnologia nunca é puramente técnica, sendo imediatamente prática, ética e política. Por outro lado, confrontada com novos desafios tecnológicos, a fenomenologia necessita assumir certas características de fenomenotecnologia; ações não são nunca meramente práticas. Uma tal combinação entre fenomenologia e fenomenotecnologia abre especiais perspectivas no que diz respeito ao trato da questão da vida humana. Primeiramente, descreveremos o eu corporal como gerador de sua própria história, na medida que evidencia graus de identidade e se constitui como altamente vulnerável. Em segundo lugar, distinguiremos entre a atitude técnica, restrita ao fato de que algo se modifica, e a atitude terapêutica, que se dirige a alguém que sofre: o paciente. A seguir, ultrapassaremos os limites do enquadramento clínico ao considerar novas conquistas no que diz respeito à produção da vida (por exemplo, clonagem e manipulação genética). Antes de simplesmente argumentar pró ou contra, temos de indagar o que significa produzir um eu vivo. Neste contexto, o tempo da poiesis será contrastado com o do pathos. Examinaremos o lugar desde o qual um ser vivo ou pessoal pode ser definido como alguém em
ABSTRACT - Certainly, as long as human beings exist, life has never been exempt from technical manipulations. However, recent forms of biotechnology go further, tending to blur the classical distinction between natural growth and artificial production. Ars sive natura, that may be the new slogan. Now, in opposition to current bioethics which try to compensate the technological monism by means of a practical dualism, we will argue that by acting upon the Other (Fremdeinwirkung) biotechnology is never purely technical, it is immediately practical, ethical and political. On the other side, faced with new technological challenges, phenomenology needs certain features of phenomenotechnology; actions are never merely practical. Such an intertwining of phenomenology and phenomenotechnology opens up special perspectives in dealing with the issue of human life. Firstly, we will describe the bodily self as generating its own history, as showing grades of selfhood and as being highly vulnerable. Secondly, we will distinguish between the technological attitude, restricted on something to be changed, and the therapeutic attitude, addressed to somebody suffering, namely the patient. In the end, we cross the limits of the clinical frame by considering new attempts of life production (e.g. cloning and gene manipulation). Before simply arguing pro and con we have to ask what does it mean to produce a living self. In this context the time of poiesis will be contrasted with the time of pathos. We will examine the place from where a living or personal being may be defined as somebody in itself, and we will simultaneously stress the

* Universidade de Bochum, Alemanha.

\begin{tabular}{|l|l|l|l|l|r} 
VERITAS & Porto Alegre & v. 51 & n. 2 & Junho 2006 & p. 159-173
\end{tabular}


si, e pretendemos tencionar simultaneamente o caráter paradoxal de atribuição de um eu ao Outro (Fremdezuschreibung), que assemelha-se ao paradoxo de ter a experiência do Outro como Outro (Fremderfahrung). Pois a pré-história e a pós-história de um alguém específico, incluindo nascimento e morte, é apenas acessível a nós em termos de substituição. Reduzidos a conjuntos de células, seres humanos seriam liberados de tudo isto. Restaria algo sobre o que falamos, mas de modo algum alguém falando a nós.

PALAVRAS-CHAVE - Biotecnologia. Terapia. Fenomenologia. Fenomenotecnologia. paradoxical character of ascribing the status of a self to the Other (Fremdzuschreibung), which resembles the paradox of experiencing the Other as an Other (Fremderfahrung). At last, the pre-history and the post-history of a oneself, including birth and death, is only accessible for us by means of substitution. Reduced to cell ensembles, human beings would get rid of all that. There would be left something we speak about, but by no means somebody speaking to us.

KEY WORDS - Biotechnology. Therapy. Phenomenology. Phenomenotecnology.

Daß menschliches Leben der Pflege bedarf, ist eine alte Einsicht. Ebenso alt ist die Einsicht, daß bestimmte Heilmittel vonnöten sind, um Krankheiten zu lindern oder $\mathrm{zu}$ überwinden. Keine Therapie ist denkbar ohne elementare Techniken, die sich Naturkräfte zunutze machen und bei Bedarf das sprichwörtliche Brennen und Schneiden ermöglichen. Doch eine Biotechnik, die das Leben im Ganzen in Angriff nimmt, ist etwas ebenso Neues wie die Bioethik, die jene gleich einem Schatten begleitet. Technologische Trends stellen uns vor die Frage, ob das gelebte Leben wirklich mehr ist als ein Residuum, das noch nicht von den diversen Biotechniken erfaßt ist.

Im Mittelpunkt der folgenden Überlegungen steht ein leibliches Selbst, das sich auf der Schwelle von Kultur und Natur bewegt. Der Mensch, dem kein fester Ort zugewiesen ist, sondern der seinen Ort immer wieder neu zu bestimmen hat, erscheint als ein Lebewesen, das nicht nur einen Logos hat und einem Ethos folgt, sondern gleichursprünglich aus dem Pathos lebt und Techniken erfindet. Folgen wir diesem Gesichtspunkt, so läßt sich das Spannungsverhältnis von Therapie und Technik entfalten, ohne daß wir einer hemmungslosen Technikeuphorie oder umgekehrt einer ängstlichen Technikphobie verfallen. Die Phänomenologie hat nur dann Aussicht, auf die technologischen Herausforderungen zu antworten, wenn sie selbst eine bestimmte Art von Phänomenotechnik einschließt. Lebenswelt und Lebenstechnik sind nicht voneinander zu trennen. ${ }^{1}$

\section{Biologie und Medizin}

Bevor Philosophen ihr Repertoire einfach um neue Titel wie 'Bioethik' oder 'medizinische Ethik' bereichern, sollten sie sich auf Anfängliches besinnen. Als Aristoteles mit der Biologie eine Lehre vom Leben schuf, betrachtete er sie als Teil

\footnotetext{
Die Voraussetzungen, von denen unsere Überlegungen sich leiten lassen, werden an anderer Stelle ausführlich entfaltet. Vgl. insbesondere Grenzen der Normalisierung (1998), Kap. 6, 9-10; Das leibliche Selbst (2000); Bruchlinien der Erfahrung (2002), Kap. VIII: "Technische Eingriffe in die Erfahrung".
} 
einer Physik, die sich mit der Physis im allgemeinen beschäftigt. Diese kosmologisch verankerte Physik erwuchs aus dem Staunen darüber, daß es Wesen gibt, die sich von selbst und aus sich selbst bewegen, ohne daß sie auf Außenkräfte, darunter die der menschlichen Techne angewiesen sind. Auch die Seele wird von Platon als etwas angesetzt, das sich aus sich selbst bewegt (Phaidros 245 d). So gibt es anfangs keine schroffe Trennung zwischen Lebewesen und Vernunftwesen. Der Logos ist schon im Bios am Werk.

Dasselbe gilt für die Medizin, doch als praktische Kunst überschreitet diese den Zyklus der theoretischen Wissenschaften, der von der Physik über die Mathematik bis zur Metaphysik reicht. Zwar stützt sie sich seit ihren hippokratischen Anfängen auf naturwissenschaftliche Kenntnisse, aber anders als die Biologie dient sie nicht der Erforschung, sondern der Heilung des Lebendigen. Doch auch dies ist nur die halbe Wahrheit. Wie uns Platon im Gorgias (463 a-c) einschärft, geht es generell um die Pflege (therapeia) des Körpers, die einerseits der Gymnastik, andererseits der Heilkunst obliegt. In der Körperpflege treffen Gesundheit und Gesundung, Heil und Heilung zusammen. Die Heilung stellt also eher eine pars pro toto dar. Heilung im spezifischen Sinne ist wichtig, aber sie ist nicht alles, daran hat sich bis heute nicht allzu viel geändert. ${ }^{2}$ Als praktische Disziplin beschäftigt die Medizin sich ferner mit Einzelfällen, selbst wenn diese sich typischen Mustern zuzuordnen sind. Der Arzt heilt Sokrates, nicht den Menschen, wie Aristoteles lapidar feststellt. Doch Heilung bedeutet alles andere als eine reine Tätigkeit von seiten des Arztes. Das deutsche Wort heilen hat ebenso wie das französische Wort guérir und ähnlich wie das spanische curar/curarse zugleich eine transitive und eine intransitive Bedeutung. Jede Therapie wäre wirkungslos, wenn sie nicht auf autotherapeutische Kräfte und auf den Heilungswunsch des Patienten zurückgreifen könnte. Darin unterscheidet sich die Heilung von einer bloßen Reparatur. Andererseits wirft jeder heterotherapeutische Eingriff in fremdes Leben rechtliche Fragen auf, da er nur unter bestimmten Voraussetzungen zulässig ist; insofern hat die Therapie hat von Anfang eine soziale oder, wie wir heute zu sagen pflegen, eine intersubjektive Dimension. Umgekehrt hat die Habeascorpusakte, die zu den ältesten Errungenschaften des Rechts gehört, in ihrer Bezugnahme auf den Körper einen medizinischen Aspekt. Medizin und Jurisprudenz greifen ineinander. Während das Recht die eigene Leibessphäre vor unerlaubten Eingriffen schützt, entscheiden medizinische Gutachten darüber, ob jemand als rechts- oder schuldfähig anzusehen ist. Das Recht errichtet eine Hürde gegen jedwede Biologisierung des menschlichen Lebens, auch wenn diese sich als Biopolitik ausgibt. Mit der Therapie geraten wir unweigerlich in Kants Streit der Fakultäten.

Die Zweiteilung der Medizin in theoretische Lebenserforschung und praktische Lebensbehandlung schließt freilich nicht aus, daß es Übergangszonen gibt. Die Physiologie gehört bis heute zum Grundstock medizinischer Lehre und Forschung.

Die Heilung als Wiederherstellung der Gesundheit bildet auch heute eher eine Ausnahme, verglichen mit medizinischen Aufgaben wie Notfallversorgung, Symptomlinderung, Minimierung chronischer Krankheiten und weitreichenden Maßnahmen wie Vorsorge und dem, was man heute Lifestyle nennt. 
Umgekehrt ist der biotechnische Eingriff in das Leben so alt wie der Werkzeuggebrauch. Man denke an alte Errungenschaften wie die Tierzähmung, die Viehzucht und den Ackerbau. Allerdings neigt die klassische Philosophie zu einer Unterbewertung der Technik, als hätte diese es nur mit lebensnotwendigen Bedürfnissen und mit dem Gebrauch von Mitteln zum rechten Leben zu tun. Der griechische Mythos dringt tiefer in die Abgründe des Lebens vor, wenn er Prometheus, der das Feuer stahl und es den Menschen überbrachte, zu einem tragischen Helden der Techne erhebt. Zur Macht der Technik gehört nicht nur der gewaltsame Eingriff in die Natur, sondern auch das listige Umgehen und Verwandeln der Naturzwänge. In seiner Phénoménologie de la perception (1945, S. 221) beschreibt Merleau-Ponty das Ineinander von Natur und Kultur mit folgenden Worten: “Tout est fabriqué et tout est naturel chez l'homme (...) en ce sens qu'il n'est pas un mot, pas une conduite qui ne doive quelque chose à l'être simplement biologique - et qui en même temps ne se dérobe à la simplicité de la vie animale, ne détourne de leur sens les conduites vitales, par une sorte d'échappement et par un génie de l'équivoque qui pourraient servir à définir l'homme." Der Traum vom Leben in reiner Natur, als die Erde, "von keiner Hacke berührt, von keiner Pflugschar verletzt, alles von selbst gab" und als sie auch die übrigen Lebewesen gebar, nachdem "die fruchtbaren Samen der Wesen, im lebenskräftigen Boden genährt, wie im Mutterleib gewachsen waren" ${ }^{3}$ führt zurück in eine goldene Vorzeit, die wir als Menschen immer schon hinter uns gelassen haben. Die Techne gehört ebenso zum Menschen wie der Logos.

\section{Biotechnik im modernen Sinne}

Allerdings wäre es abwegig, anzunehmen, die moderne Technik habe es immer schon gegeben - und als liefere die Philosophie der Technik insgesamt nur Fußnoten zu Aristoteles. Biotechnik im modernen Sinne fällt in eine späte Phase der Technikgeschichte. Grob gesehen lassen sich drei technologische Paradigmen unterscheiden, die jeweils von einem bestimmten Verhältnis des Menschen zur Natur dominiert werden (vgl. Waldenfels 2002, S. 362-374). Das klassische Paradigma zeichnet sich aus durch eine Einbindung der Technik in die Natur; alles wird zum eigenen Gebrauch hergestellt unter Verwendung geeigneter Werkzeuge wie Hammer und Gefährt. Das moderne Paradigma kulminiert in der Beherrschung der Natur durch Technik; es werden automotorische Maschinen wie Mühlrad oder Windmühle eingesetzt zur Nutzung fremder Kräfte. Davon zu unterscheiden ist ein hypermodernes Paradigma; mit der Entfesselung der Technik als Quasi-Natur treten Prozesse der automatischen Selbstregelung und der Selbstorganisation in den Vordergrund. Auf diese Weise erhöht sich die Reichweite der Technik in einem Ausmaß, dessen Grenzen noch nicht abzusehen sind. ${ }^{4}$

Die Fragen, die heute unter den Rubriken von Biotechnik oder Biomedizin verhandelt werden, fallen vorwiegend unter das dritte der erwähnten

3 Ovid, Metamophosen I, 101 f., 416-421, dt. Übersetzung M. v. Albrecht.

Vgl. dazu vom Verf. Der Stachel des Fremden (1990), Kap. 9: "Reichweite der Technik". 
Paradigmen. So geht es nicht um eine schlichte Herstellung des Lebens, sondern um eine besondere Art von Poiesis, in der sich Züge einer Autopoiesis mit denen einer Heteropoiesis verbinden. ${ }^{5}$ Es zeichnet sich eine Tendenz ab, die darauf hinausläuft, den herkömmlichen Unterschieds zwischen natürlicher Entstehung und künstlicher Herstellung zu neutralisieren. Diese Tendenz läßt sich in die Formel fassen: ars sive natura, und dies mit einem durchaus theologischen Unterton, in dem das Erbe des Deus faber anklingt. Die Biopoiesis kann sich wie im Falle der Transplantation auf bestimmte Körperorgane beschränken, damit läge sie auf der Linie einer biologischen Schrift wie De partibus animalium; sie kann aber auch auf das Lebensganze ausgreifen wie im Falle der künstlichen Befruchtung oder des reproduktiven Klonens, damit läge sie auf der Linie einer Schrift wie De generatione animalium. Dabei gilt es zu beachten, daß wir es bei den korrespondierenden Formen der Bioethik und Biomedizin mit keiner bloßen Ergänzung herkömmlicher Disziplinen und keiner bloßen Erweiterung des Fächerkanons zu tun haben; vielmehr bemüht sich die Bioethik um eine Kompensation jener technischen Neuerungen, die von der Biotechnik ausgehen und sich zu einer allgemeinen Biopolitik und Bioökonomie ausweiten. Die Entnahme von Organen zwingt zur Präzision des Todeszeitpunkts, die künstliche Befruchtung erfordert Regelungen darüber, wie mit überzähligen Zellen umzugehen ist, und die Möglichkeit genetischer Eingriffe in das menschliche Erbgut wirft die Frage nach einer entsprechenden Verfügungsgewalt auf. ${ }^{6}$

Damit stellt sich für uns die Frage, was es mit den Möglichkeiten einer Lebensherstellung auf sich hat und was überhaupt unter Lebensherstellung zu verstehen ist. Zunächst einmal stoßen wir auf einen praktischen Dualismus, den Auswirkungen weit über den theoretischen Dualismus von Leib und Seele, von Körper und Geist hinausgehen (vgl. Waldenfels 2002, S. 427-433). Wir haben es nicht mehr bloß mit zweierlei Substanzen zu tun wie in der theologisch abgestützten cartesischen Ontologie und auch nicht bloß mit zweierlei epistemischen Einstellungen, die sich in verschiedenartigen Beschreibungssprachen artikulieren, vielmehr kommt es zu einem Auseinanderklaffen heterogener Praktiken. Kantisch betrachtet begegnet uns der Mensch als Bürger zweier Welten. Einmal tritt er auf als Lebewesen, dessen Verhaltens- und Erlebnisweisen von Natur aus determiniert und durch technische Eingriffe veränderbar sind, wie es uns der Behaviorismus vorexerziert

Unter Poiesis versteht Platon im allgemeinen die "Ursache dafür, daß etwas aus dem Nichtsein in das Sein tritt" (Symposion 205 b). Zur Autopoiesis vgl. die bekannten Forschungen von Humberto R. Maturana.

6 Vgl. dazu aus jüngster Zeit die praktisch-technischen Überlegungen des Mediziners Klaus Dörner: Der gute Arzt (2001), S. 297-300, die medizin-soziologischen Untersuchungen von Gesa Lindemann: Die Grenzen des Sozialen. Zur sozio-technischen Konstruktion von Leben und Tod in der Intensivstation (2002) sowie zum zweifelhaften Status der neueren Bioethik und Biotechnik Petra Gehring, Was ist Biomacht? (2006). 
hat. Das andere Mal tritt er als Vernunftwesen auf, das sich als frei erweist, indem es Zweck an sich selbst ist und eigenen Gesetzen folgt, und das uns als solches eine moralische Achtung abverlangt. Doch eine säuberliche Aufspaltung der Erfahrung gemäß heterogener Seins- und Erkenntnisweisen läßt sich nicht länger durchhalten, sobald es zu Fremdeinwirkungen kommt, die beide Reiche, das Reich der Natur wie das der Freiheit, durchqueren. Parodistisch formuliert sähe eine etwaige Aufspaltung wie folgt aus: Als Naturwesen mache ich mit dir (genauer: mit ihm), was ich will, als Vernunftwesen achte ich dich. Doch könnte man beides auseinanderhalten, so wäre die Einwirkung keine Fremdeinwirkung, die Fremdbeziehung keine Fremdeinwirkung. Sachbezug und Fremdbezug würden getrennte Wege gehen. Man würde das Problem lösen, indem man das problematische Phänomen der Fremdeinwirkung in Nichts auflöst. Doch dem steht entgegen, daß eine Biotechnik, in der Fremdeinwirkungen eine konstitutive Rolle spielen, unmittelbar praktisch, ethisch, politisch ist; denn sie bezieht sich von vornherein nicht bloß auf etwas, das in der Welt vorkommt, sondern auf jemanden, der/die selbst eine Welt hat und von dem/der ich selbst angerührt, angeblickt, angesprochen werde. Die fremden Ansprüche, die hier auftreten, lassen sich verdrängen, aber nicht austilgen. Inmitten der Physiotechnik öffnet sich ein Raum für Lebensdebatten, die es nicht gab, solange die Naturtechnik sich auf die Beeinflussung physikalischer und chemischer Prozesse beschränkte.

Für Philosophen und Theologen stellt sich die Frage, wie sie auf solch neuartige Herausforderungen reagieren sollen. Die landläufigen Antworten fallen sehr gegensätzlich aus. Auf der einen Seite flüchtet man sich in einen Konformismus. Man läßt sich die entscheidenden Begriffe von den sogenannten Biowissenschaften vorgeben und reagiert mehr oder weniger kasuistisch, sobald ein neues Problem anfällt, so etwa, indem man den Tod als Hirntod definiert und umgekehrt Lebenspässe ausstellt wie ein biologisches Standesamt. Eine derartige Bioethik wäre nichts weiter als eine Ethik in usum scientiae, die sich auf Zubringerdienste beschränken würde. Auf der anderen Seite neigt man zur Prinzipienreiterei. Man verläßt sich auf große Instanzen wie Freiheit, Würde, Vernunft, die nahezu tautologisch definiert und von aller fremden Einmischung freigehalten werden. Vernunft und Moral sind dann etwas, das der Mensch hat, sofern er ein Vernunftwesen ist. Wir fühlen uns auf einem sicheren Boden. Am Ende hätte der Personenstatus gar nichts mit Biologie zu tun hat, so bei Robert Spaemann, der Jemand und Etwas strikt auseinanderhält. Prinzipiell Gesinnte gehen ebenso wie konformistisch Gesinnte von vorgefaßten Ansichten aus und nicht von der Sache selbst, die sich als viel verwickelter und bedrohlicher darstellt.

Vgl seinen Beitrag "Wer jemand ist, ist es immer", in Geyer 2001, hier S. 79. 


\section{Phänomenologie des leiblichen Selbst}

Den Begriff des Selbst (ipse) verwende ich in strikter Abgrenzung von dem des Selben (idem). Das Selbe gehört seit Platons Sophistes zu den Grundkategorien, mit denen wir etwas als etwas von etwas anderem unterscheiden. Prozesse der Identifikation, einschließlich der Selbstidentifikation, sind auch auf Lebewesen anwendbar, doch sind sie nicht konstitutiv für das, was das leibliche Selbst als es selbst ausmacht. Das Selbst entspringt einem Prozeß der Selbstabgrenzung. Anders als das Unterscheiden von etwas fällt das originäre Sichunterscheiden in die Teilnehmerperspektive und nicht in die Beobachterperspektive. Erst im nachträglichen Vergleich, der beide Perspektiven miteinander verschränkt, erscheint der Freund als ein anderes Selbst, als ein heteron auton (Nik. Ethik IX, 9), das Ich als ein alter ego. Das Selbst, das als Ichselbst und als Du-selbst auftritt, bildet den Kern einer Eigen- und Binnensphäre, in Abhebung vom Fremden und Äußeren, das auf lateinisch extraneus heißt. Der Selbstbezug, der das Selbst charakterisiert, taucht in verschiedenen Formen auf: als Selbstgefühl in Lust und Schmerz, als Selbstbeherrschung, Selbsterkenntnis, Selbstsorge, auch als Selbsttechnik und im äußersten Fall als Selbsttötung. Jeder Selbstbezug schließt einen Selbstentzug ein. Wie alle Erfahrung so beginnt auch die Selbsterfahrung anderswo, ursprünglich äußert sie sich als Selbstaffektion, nicht als Selbstsetzung. ${ }^{8}$ Das Selbst weiß von sich nur aus der Ferne. Wer sich selbst im Spiegel erblickt, sieht sich selbst in einem fremden Medium, in Form einer gebrochenen Identität, und ähnliches gilt für andere Weisen des Selbstbezugs. Fremdheit erfahren wir ursprünglich am eigenen Leib.

Ein Selbst, das nicht einfach bei sich ist, sondern nur immer zu sich kommt, verfestigt sich zu keiner identischen Substanz, es durchläuft einen stetigen Prozeß des Selbstwerdens und Selbstentwerdens. ${ }^{9}$ Dieser Prozeß setzt sich nicht aus jenen diskreten Akten und Zuständen zusammen, die man seit John Locke zur Beschreibung einer subjektiven Innenwelt heranzuziehen pflegt, wobei man innere Teilnahme und äußere Beobachtung zur hybriden Mischung einer Introspektion verschmilzt. Die Sprache der Biotechnik und auch die der Bioethik sind bis heute weithin von dieser Auffassung geprägt. Prozesse des Erwachens und Einschlafens, des Geborenwerdens und Sterbens und auch die des Alterns werden an einer Entwicklungsskala gemessen, die an das Leben angelegt und nicht aus dem Leben selbst gewonnen wird. Hinzu kommt, daß ein Selbst, das es selbst wird und nicht einfach es selbst ist, Modi des Selbstseins zuläßt. Man ist mehr oder weniger man selbst. Diese Graduierung widerspricht schroffen Antithesen wie der von Person und Sache, die dazu führen, daß sich zwischen der menschlichen Welt und der Welt der Tiere und Pflanzen Klüfte auftun.

8 Würde man von einer puren Selbstsetzung ausgehen, so würde man auf die bekannten Aporien stoßen, denen ein reflexiv gefaßtes Selbstbewußtsein ausgesetzt ist.

Vgl. im Französischen den Unterschied von se faire und se défaire, dessen sich Bergson bedient. 
Natürlich stellt sich die Frage, wie das menschliche Selbst sich aus dem Bereich des Lebendigen heraushebt. Halten wir uns an die genannte Eigenart eines Selbst, so können die Unterschiede zwischen Mensch und Tier wiederum nur aus einer Selbstabgrenzung gewonnen werden. Das menschliche Selbst erfährt sich nicht dadurch als etwas Besonderes, daß zum Status des Lebewesens etwas Anderes, Höheres hinzutritt, sei es der Logos als Vernunft und Rede, sei es die Freiheit, sei es eine unsterbliche Seele. Was den Menschen auszeichnet, ist vielmehr - mit Nietzsche zu reden - der Status eines "nicht festgestellten Tieres", der ihn permanent vor Fragen stellt wie diese: Wer bin ich? Wer bist du? Wo sind wir? Was sollen wir tun? Dies besagt, daß die Differenz zwischen Innen und Außen, zwischen Eigenem und Fremdem, zwischen Menschlichem und Tierischem innerhalb unserer selbst auftritt, so wie die Differenz zwischen Kultur und Natur innerhalb der Kultur auftaucht. Somit lebt das menschliche Selbst auf der Schwelle von Innen und Außen, in gleichzeitiger Nähe und Ferne zu sich selbst, also auch in Nähe und Ferne zu allem Lebendigen. In seiner anthropologischen Schrift Mutmaßlicher Anfang der Menschengeschichte bemerkt Kant, der Mensch stehe seit seiner "Entlassung aus dem Mutterschoße der Natur" gleichsam "am Rande eines Abgrundes"; die "Unendlichkeit" an Möglichkeiten, die sich seinem Begehren auftut, nötigt ihn also, sich selbst selektiv festzulegen. Dabei sieht sich der Mensch wechselnden Herausforderungen ausgesetzt, die ihm jeweils verschiedenartige Antworten abverlangen. Was wir Kulturen nennen, sind Antworten en gros, und zwar erfinderische Antworten, die nirgends vorprogrammiert sind, außer in der Form von Skizzen, die gewisse Antworten nahelegen, aber keine bestimmte Antwort erzwingen. Diese Plastizität entspricht der Annahme einer verzweigten Evolution, die nicht auf einer einzigen Bahn bewegt, und sie entspricht auch neurologischen Modellen, die mit Netzwerken und Parallelschaltungen operieren.

Als leibliches Selbst ist das lebendige Selbst schließlich ein verletzliches Selbst. Verletzlichkeit setzt ein bestimmtes Maß an Integrität voraus. Ein Wesen, das sich selbst nach außen hin abgrenzt und das als menschliches Selbst zugleich um seine Grenzen weiß, zeichnet sich aus durch eine Verletzlichkeit, die über die Möglichkeiten bloßer Sachbeschädigung weit hinausgehen. Ein "denkendes Schilfrohr", das sich seines heiklen Status bewußt ist, bedeutet mehr als bloßes Gras, das zertreten wird und verwelkt. Damit stellt sich die Frage nach den Grenzen, denen Eingriffe in das Leben unterliegen, und diese Frage stellt sich umso dringlicher, je mehr diese Eingriffe bis zum Kern des lebenden Selbst vordringen.

\section{Therapeutische und technische Einstellung}

Das Verhältnis von Therapie und Biotechnik spiegelt sich wider in der Gegensätzlichkeit zweier Grundeinstellungen, die komplexer ist als das Gegensatzpaar von naturalistischer und personaler Einstellung, wie wir es aus der Phänomenologie Husserls kennen (vgl. 1952, § 49). Die therapeutische Einstellung 
beruht auf einer triadischen Beziehung. Der Therapeut behandelt etwas: Leiden, Störungen, Schmerzen, und er behandelt zugleich jemanden: den Patienten, der unter etwas leidet. Die Therapie nimmt als Therapie responsive Züge an, sofern sie auf fremde Ansprüche antwortet, selbst wenn ihre Antworten immer wieder in technischen Apparaturen zu versickern drohen. Als Fremdeinwirkung trifft die Therapie stets auf einen Adressaten, an den sie sich richtet, und nicht nur auf einen Referenten, auf den sie sich bezieht. Auch Körperverletzung und Tötung sind Formen eines An-tuns, kein bloßes Tun und Machen. Die technische Einstellung beruht dagegen auf einer dualen Beziehung. Der Techniker, mitsamt den technischen Apparaturen, die er einsetzt, bezieht sich auf etwas: auf einen Körperprozeß oder einen Körperzustand, auf den er direkt einwirkt, etwa durch Herzoperationen oder Gewebeentnahme, oder auf Prozesse der Selbstregulierung oder Selbstorganisation, an denen er indirekt mitwirkt, etwa durch die Verabreichung von Medikamenten oder durch neurochirurgische Eingriffe.

Der Zusammenhang, der sich zwischen diesen beiden Einstellungen ergibt, zeigt gewisse Ähnlichkeiten mit der Verfahrensweise der Ethnologie, sofern man darunter eine besondere Art der Wissenschaft versteht, die sich in Form einer Xenologie mit dem kulturell Fremden beschäftigt (vgl. Waldenfels 1997, Kap. 4). Wir sind es gewohnt, der Teilnehmerperspektive, die in der Sprache der ersten und zweiten Person ihren Ausdruck findet, die Beobachterperspektive gegenüberzustellen, deren Feststellungen und Erklärungen in der dritten Person formuliert werden. Doch eine derartige Alternative wird der ethnologischen Ausgangslage nicht gerecht; diese erfordert eine teilnehmende Beobachtung, die sich nicht völlig von dem sozialen Feld ablöst, innerhalb dessen die Forschung stattfindet. In jedes Interview und in jede Aufzeichnung geht etwas von der Fremdheit ein, auf die es die Forschung abgesehen hat. Ähnliches trifft zu auf die Betätigungen des Mediziners, die es mit der Krankheit als einer spezifischen Form der Fremdheit zu tun haben und nicht mit einem bloßen Defizit, mit einer anderen Ordnung und nicht mit einer bloßen Unordnung. ${ }^{10}$ Eine responsive Art von Therapie, die sich an Adressaten wendet und nicht bloß auf Bezugs- und Zielobjekte ausgerichtet ist, stellt sich dar als ein Ineinander von Therapie, Theorie und Technik. Nur so wird aus der Technik eine Heiltechnik.

Ansatzpunkt für eine indirekte Technik, die an jemandem ausgeübt wird, ist der Leib des Patienten. In seiner Doppelrolle als Leibkörper ist der Leib nicht nur lebendiger Ausdruck all unserer Intentionen und Affektionen, sondern ihm haftet auch eine eigentümliche Materialität an, die in der Ermüdung als Nachlassen der eigenen Kräfte oder im Schmerz als Einwirkung widriger Kräfte erfahren wird. Diese Doppelheit, die nicht mit der Dualität getrennter Seins- oder Erkenntnisbereiche zu verwechseln ist, bezeichnet Husserl mit einem treffenden Ausdruck als "Umschlagstelle" (1952, S. 286). In unserem Leib gehen Kultur und Natur, Geist und Natur, Persönliches und Anonymes, Eigenes und Fremdes

${ }^{10}$ Vgl. dazu ausdrücklich das Kapitel "Der Kranke als Fremder. Therapie zwischen Normalität und Responsivität" in: Grenzen der Normalisierung (1998). 
fortwährend ineinander über. Die Fremdheit, die dem eigenen Leibes anhaftet, bietet Ansatzpunkte für eine technologische Epoché, durch die Selbstbezüglichkeit des eigenen Leibes und die konkrete Lebensbedeutsamkeit des leiblichen Geschehens systematisch ausgeblendet wird. Mittels dieser Epoché reduziert sich der Patient auf den Status eines anatomischen Etwas, das aus Blutgefäßen, Organen und Zellgeweben besteht, und sein Erleben reduziert sich auf meßbare Prozesse wie Erhöhung des Blutdrucks, Ausschüttung von Hormonen oder das Feuern von Neuronen. Diese Reduktion entspricht der naturalistischen Einstellung bei Husserl, nur daß sie hier - wie in jeder experimentellen Wissenschaft - ganz und gar mit einer technischen Einstellung verquickt ist. Allerdings besteht immerzu die Gefahr, daß die Technologie sich verselbständigt und daß die Lebensfäden, die der Biotechnik im Gegensatz zur bloßen Physiotechnik anhaften, sich verdünnen. Die Klinik bildet einen institutionellen Rahmen, der verhindert, daß die Heiltechnik sich völlig von der Therapie ablöst. Der Chirurg würde wegen Körperverletzung belangt, sobald er Eingriffe vornähme, die diesen Rahmen mißachten. Doch jede Institution, auch die Klinik, ist von Randzonen umgeben, in denen hybride Gestalten ihr Spiel treiben, so in der Degradierung des Patienten zum Versuchsobjekt, in der Verwandlung von Medikamenten in verkäufliche Präparate oder im Handel mit Transplantationsorganen. Das Wort Pharmaindustrie ist so zweideutig wie die Sache, die es benennt. Das Ineinander von Therapie, Ökonomie und Technik erzeugt Spannungen, für die es keine Patentlösung gibt, die aber nach einer besonderen therapeutischen Wachsamkeit verlangen.

\section{Biotechnische Perspektiven}

Die jüngsten Entwicklungen der Biotechnik lassen neue Fragen aufkommen, die über den Rahmen einer Heiltechnik weit hinaus zielen. Ältere Formen einer Eugenik, die wir sie schon bei Platon finden, erwachen zu neuem Leben, nur daß diese neuerliche Eugenik nicht mehr an einer vorgegebenen Natur Maß nimmt, die noch bei Paracelsus als "große Apotheke" wirkt, sondern an einer manipulierbaren Natur. Hätte diese das letzte Wort, so würde die Lebenskunst einer schrankenlosen Biotechnik überantwortet. Doch da wir als "nicht festgestellte Tiere" immerzu mehr tun können, als wir tun wollen oder tun sollen, lassen sich praktische Fragen niemals rein technologisch beantworten. Jede technologische Vision lebt von Präferenzen, die nicht selbst technologisch erzeugt wurden. Die Tatsache, daß etwas wohl funktioniert, beantwortet nicht die Frage, warum es besser ist, daß etwas so geschieht und nicht anders. ${ }^{11}$ Heideggers schlichte Feststellung, das Wesen der Technik sei ganz und gar nichts Technisches (1954, S. 13), läßt sich bis heute nicht von der Hand weisen.

\footnotetext{
11 Vgl. dazu Platons Zweifel an einer Rechtfertigung des menschlichen Verhaltens aus rein natürlichen Ursachen (Phaidon 98 b-99 d). Allerdings reicht es nicht aus, diese als bloße Formen einer conditio qua non zu behandeln. Die Mitwirkung der Natur, die im Falle meines Leibkörpers durchaus als "meine Natur" zu bezeichnen ist (Husserl 1952, S. 280), bedeutet mehr als die Bereitstellung notwendiger Bedingungen.
} 
Nehmen wir die derzeit übliche Parzellierung des werdenden Lebens. Die neuere Embryologie unterscheidet zwischen Entstehungsphasen wie Vorkernstadium nicht befruchteter Eizellen; Zwei-Zell-Stadium der Zyote; Acht-ZellStadium, das eine Präimplantationsdiagnose erlaubt; Stadium der Blastozythen, das die Entnahme embryonaler Stammzellen zuläßt; schließlich das Stadium der Nidation, in dem ein individuelles Embryo entsteht. Diese "Befruchtungskaskade", mit der Embryologie entstammt, stellt den Gesetzgeber vor die Entscheidung, ob er womöglich sämtliche Stadien schützen oder lediglich eine "abgestufte Schutzwürdigkeit" statuieren soll. ${ }^{12}$ Doch unabhängig davon, wie diese Entscheidung im einzelnen ausfallen mag, gibt die zeitliche Bemessung der Phasenentwicklung zu eigenen Fragen Anlaß. So fragt es sich, ob für die künstliche Erzeugung lebendiger Individuen nicht ähnliches gilt wie für therapeutische Eingriffe in die Lebensvorgänge; dies hieße dann, daß man kein bloßes Etwas produziert, sondern Jemandem dazu verhilft, auf die Welt zu kommen. Die übliche Geburtshilfe würde auf diese Weise vorverlegt, indem sie die neuartige Form einer Erzeugungshilfe annähme. Das klingt nach einem bloßen Verbalismus. Doch für Phänomenologen, die von der Sache selbst ausgehen und nicht von bloßen Meinungen über die Sache, kann die erste Frage nicht lauten: "Sollen oder dürfen wir Lebendiges fabrizieren?", sie muß vielmehr lauten: "Was kann Lebensherstellung überhaupt heißen?" Was unterscheidet den technisch erzeugten Menschen aus der Retorte von traditionellen Phantomgebilden wie dem Homunkulus oder dem Golem? Die auffällige Tatsache, daß die Filmleinwände inzwischen von solch künstlichen Gestalten wimmeln, weist hin auf entsprechende Wunsch- und Angstvorstellungen, aber eine Lösung der Lebensrätsel ist darin nicht zu finden. Es gibt Indizien dafür, daß hier etwas nicht stimmt. ${ }^{13}$ Der Umstand, daß man im Falle des Klonens therapeutische Ziele in Anspruch nimmt (bisweilen vielleicht auch nur vorschiebt), um technische Eingriffe in die Lebenssubstanz zu rechtfertigen, läßt darauf schließen, daß man diese nicht als rein technisch betrachtet; denn dann wäre eine Rechtfertigung überflüssig. Man könnte klonen, so gut und so viel man wollte. Ein Technikvisionär wie Ray Kurzweil träumt denn auch von einem therapeutischen Klonen, das es uns erlaubt, "unseren Körper zu verjüngen, ihn fast unendlich funktionsfähig zu halten". "Wäre umgekehrt die Nutzung von Embryonen von mehr als bloß technischer Art, hätte sie es mit werdendem Leben zu tun und nicht mit bloßen Lebensmaterialien, so müßte die verbrauchende Embryonenforschung, in der es vorkommen kann, daß ein werdendes Kind als "Lieferantenkind" für ein anderes Kind dient, indem es für dessen Behandlung Stammzellen zur Verfügung stellt, als eine Form von Lebenskonflikt gefaßt werden. Dieser gliche der Abtreibung bei Lebensgefährdung

12 Vgl. dazu unter Bezugnahme auf das deutsche Recht den Biologen und Embryologen Henning M. Beier: "Unterschiedliches Recht für menschliche Embryonen", in: Frankfurter Allgemeine Zeitung vom 30. 8. 2000.

13 Bezüglich der Eingriffe in das menschliche Erbgut wären ähnliche Erwägungen anzustellen; wir lassen diese beiseite, da sie über den Zusammenhang von Therapie und Technik weit hinausgehen.

So in einem Gespräch, das in der Frankfurter Allgemeinen Zeitung vom 5. 7. 2000 erschien. 
der Frau oder der Tötungserlaubnis im Krieg, auf keinen Fall ließe er sich in einer bloßen Nutzen-Kosten-Rechnung verbuchen. Der Ausdruck "therapeutisches Klonen”, der sich einzubürgern beginnt, hat etwas Mißverständliches. Er suggeriert, das Klonen sei selbst eine Form der Therapie. Genauer müßte es jedoch heißen: "Klonen zu therapeutischen Zwecken". Dann aber stellt sich die weitere Frage: "Klonen zu wessen Heilungszweck?" In den Fällen, wo einem Patienten eigene adulte Zellen entnommen werden, stellt sich diese Frage nicht, da der technische Eingriff an eben dem vorgenommen wird, dem dieser Eingriff zugute kommt; auf diese Weise bliebe der technische Vorgang in das therapeutische Verfahren eingebettet. Beim Verbrauch fremder Embryonen ist dies nicht der Fall. Hier klaffen Nutzobjekt und Nutznießer auseinander wie im Fall des Sklaven, der laut Aristoteles "eines anderen ist" (Met. I, 982 b 26) und der als "beseeltes Werkzeug" (Politik I, 4) nicht selbst an den Zielen partizipiert, denen seine Verrichtungen dienen.

\section{Das umstrittene Selbst}

Unsere Überlegungen tendieren zu der Annahme, die Verletzung von Ansprüchen eines leiblichen Selbst stelle ein ethisches, rechtliches und politisches Problem dar, das sich mit rein technologischen Erwägungen nicht erledigen läßt. Jedes in seinen Eigenansprüchen verletzte Selbst stellt den, von dem die Verletzung ausgeht, auf direkte oder indirekte, auf ausdrückliche oder unausdrückliche Weise in Frage. Der Adressat der Verletzung ist mehr als ein Etwas, dessen Beschädigung man als Benutzer oder Beobachter beklagen mag. Sobald jemand verletzt ist, geht die Klage von ihm selbst aus, selbst wenn sie wie im Falle unmündiger Wesen nur stellvertretend erhoben werden kann. Dieser entscheidende Sachverhalt wird verdunkelt, wenn der Werdegang des leiblichen Selbst in eine Abfolge diskreter Zustände aufgelöst und auf einer linearen Zeitachse verzeichnet wird, zunächst in Form einer Befruchtungskaskade, die sich in Tagesfristen berechnet, und anschließend in einer Entwicklung des Embryos, deren Phasen sich nach Monaten bemessen. Wer diese Genese vor seinen Augen ablaufen läßt, findet sich genötigt, irgendwann eine Zäsur anzusetzen, wo etwas, über das wir mehr oder weniger verfügen und das wir möglicherweise fabrizieren, zu jemand wird, der oder die sich selbst spürt und uns dereinst von sich aus anblicken und anreden wird. ${ }^{15}$ Geht man geradewegs von einer Zäsur aus, die ein Etwas von einem Jemand trennt, so erscheint der Übergang von der Zelle zur Person als eine moderne Form der Urzeugung, als eine generatio aequivoca. Theorien der Emergenz machen die Sache nicht verständlicher, solange sie sich auf das Auftreten neuer Eigenschaften und Zustände beschränken. ${ }^{16}$ Gehen wir

15 Die Geschlechterdifferenz, die sich hier andeutet, enthält einen Hinweis darauf, daß sich jemand von sich aus auf einen Anderen bezieht und nicht bloß mit alternativen Merkmalen auftritt wie ein männliches oder weibliches Chromosom.

16 Bezieht man sich auf Prozesse der Selbstorganisation, so bleibt das Selbst dieser Organisationsweisen zu befragen. Ist das, ich selbst' oder, du selbst ' lediglich eine Spielart des systemischen, es selbst'? Vgl. dazu vom Verf. Bruchlinien der Erfahrung (2002), S. 416 f. 
umgekehrt davon aus, daß der Leib als Umschlagstelle zwischen selbsterlebtem Pathos und äußerer Kausalwirkung fungiert, so entdecken wir an ihm selbst zwei verschiedene Formen der Zeitlichkeit, die sich nicht miteinander decken: eine lineare Zeit, in die etwas als früher oder später eingeordnet wird, und eine diastatische Zeit, ein Auseinandertreten, im Zuge dessen jemand sich selbst vorausgeht, sich selbst überrascht, so daß die Betroffenen, paradox gesprochen, älter sind als sie selbst (vgl. Waldenfels 2002, S. 54-60). Als werdendes und gewordenes Selbst durchläuft das Selbst eine pathische Zeit der Widerfahrnisse, die sich deutlich von der poietischen Zeit des Machens unterscheidet, so daß jeder evolutionäre Monismus ausscheidet. Das Selbst verweist auf eine Vorzeit, die immer schon begonnen hat und die es immer schon verwickelt ist, wenn der Zeitablauf in verschiedene Zeitstrecken zerlegt und mit zeitlichen Indices versehen wird. Das Selbst hat eine Vorgeschichte, die in der Zuschreibung von Eigenschaften, Zuständen und Vorgängen bereits vorausgesetzt ist. Das Selbst, das sich auf elementare Weise in Lust und Schmerz, im Wohlbefinden und Mißbefinden bekundet, entpuppt sich als das Unzuschreibbare in allem Zuschreiben.

Eine Ethik, die sich nicht auf bloße Dienstleistungen für eine biologische Form der Techno-Science zu beschränken gewillt ist, sieht sich mit zwei grundlegenden Fragen konfrontiert. Zum einen stellt sich eine Begründungsfrage: Gibt es moralische und rechtliche Gründe, die den bloßen Gebrauch und Verbrauch eines leiblichen Selbst ausschließen? Zum anderen stellt sich eine Definitionsfrage: Welche Wesen sind mögliche Kandidaten für ein zu achtendes und zu schützendes Selbst, und welche Lebensphasen und Lebenszustände kommen dabei in Betracht? Die erste Frage bedürfte einer langen Debatte. Pragmatiker geben sich nicht selten mit Common Sense-Argumenten zufrieden, indem sie sich auf tradierte Werte, religiöse Überzeugungen oder auf Verfassungsgrundsätze berufen. Philosophen können nicht umhin, solche Begründungsweisen selbst in das sachliche Fragen einzubeziehen, indem sie nicht nur nach der Begründungsart, sondern auch nach der Begründungsfähigkeit moralischer Grundsätze fragen. ${ }^{17}$ An dieser Stelle begnüge ich mich mit einigen abschließenden Bemerkungen zur zweiten Frage nach der Definition eines Selbst, das uns mit eigenen Ansprüchen entgegentritt.

Zunächst gilt es das Fragefeld zu umreißen. Die Frage nach den Unterscheidungskriterien, die der Bestimmung eines achtungs- oder schützenswerten Wesens zugrunde zu legen sind, kann nicht am Anfang stehen. Zuvor fragt es sich, wer von welchem Ort aus solche Definitionen aussprechen und entsprechende Zuschreibungen vornehmen kann. Die Annahme, relevante Zuschreibungen ließen sich aus einer reinen Beobachterperspektive gewinnen, die

\footnotetext{
Ich selbst beharre auf einem, blinden Fleck' der Moral, der es ausschließt, daß Moral sich selbst begründet; was über eine jede Gesetzesmoral hinausgeht, wäre eine responsive Ethik, die aus fremden Ansprüchen entspringt. Ich verweise diesbezüglich auf meine Ausführungen in Schattenrisse der Moral (2006).
} 
sich problemlos mit der technischen Einstellung verbindet, scheidet aus. Die Erfassung des Selbst als eines Selbst entspringt nämlich einer Form der Fremdzuschreibung, die als Zuschreibung des Unzuschreibbaren nicht minder paradox ist als die Fremderfahrung, die es laut Husserl mit der Zugänglichkeit des original Unzugänglichen zu tun hat. Würden ich dem Anderen das Selbst eigenmächtig zuschreiben, so wäre es kein fremdes Selbst, das mir entgegenkommt und mich Anspruch nimmt; das Selbst wäre von mir eingesetzt und könnte von mir wieder abgesetzt werden.

Ausgangspunkt einer solchen Fremdzuschreibung ist ein aktuelles, normal entwickeltes Selbst, das mit anderen Selbsten im Konnex steht. Es muß soweit entwickelt sein, daß es die Frage nach der Achtung des Selbst überhaupt aufwerfen kann. Weder Kinder noch Föten, weder Schlafende noch Sterbende sind dazu in der Lage. Dies bedeutet, daß man nicht von Anomalien, von Grenz- und Ausnahmefällen ausgehen kann, als wären dies einfache Tatsachen, die für sich selbst sprechen. Anomalien sind nur von einer vorgängigen Normalität her zu fassen.

Dieser Ausgangspunkt der Zuschreibung betrifft einmal die schon erwähnte Vor- und Nachzeitigkeit. Die Geburt gehört zur Vorgeschichte eines Selbst, der Tod zu seiner Nachgeschichte. Darüber hinaus kommt es im Schlaf, in der Ohnmacht oder auch im Wachkoma zu einer Unterbrechung der wach durchlebten Geschichte. Die Vorgeschichte umfaßt all das, was zu früh kommt und nicht bloß relativ früher als die Antwort, die der Betroffene darauf gibt. In diesem Sinne spricht Husserl von einem Vor-Ich, Freud von einer kindlichen und kollektiven Vorzeit. Wir haben es mit Zeitverschiebungen zu tun und nicht mit der bloßen Sukzession von Zeitphasen. Des weiteren sind Wesen, die auf die Welt kommen, in eine Mitwelt eintreten und diese am Ende wieder verlassen, auf Stellvertretung angewiesen, nämlich auf Fürsprache und Fürsorge. Was in der Vor- und Nachgeschichte oder in den Höhlungen der Geschichte geschieht, bringen jeweils Andere zur Sprache und nicht jene, denen dies geschieht. Kinder treten in die Welt, indem sie erwartet, befürchtet oder aber verhindert werden; Tote verlassen die Welt, indem sie betrauert, verabschiedet, erinnert oder aber verscharrt und vergessen werden. Geburts- und Sterberituale, die zur Interpretation des Lebens, aber zugleich zu dessen Rändern gehören, verweisen darauf, daß jede Fremderinnerung auf Unerinnerbares, jede Fremderwartung auf Unerwartbares stößt.

Eine rein technische Einstellung zu Leben und Tod würde keine Geschichte zulassen: ein Zellhaufen kennt nur vorhergehende und nachfolgende Zustände. Die rein technische Einstellung würde auch keine Stellvertretung zulassen: für Vorgänge in einem Zellhaufen kann niemand einstehen. Die Teilhabe an einer gemeinsamen Welt, die Aristoteles als Mitleben, Heidegger als Mitsein bezeichnet, wäre unterbunden. Außerdem müßte ich als Technikbesessener bereit sein, diese Einstellung auf mich selbst anwenden. Ich müßte Geschichten erzählen wie: "Als ich noch ein Zellhaufen war". Diese Geschichten wären rein fiktiv, da jede erzählte Geschichte einen Selbstbezug dessen voraussetzt, der sie durchlebt 
hat. Die 'Sache des Lebens' ist nicht zu denken, ohne daß die Lebenstechnik an sich hält. Ohne Schonung des Lebendigen gibt es für uns kein Lebendiges.

Denkt man das Leben von seinen Steigerungsformen und nicht von seinem Niedergang her, so beginnt es damit, daß uns etwas widerfährt. Dazu gehören Stiftungen jeder Art, darunter wissenschaftliche Entdeckungen und technische Erfindungen. Dies besagt, daß jedes eigene Wort eine Antwort auf Fremdes, jeder eigene Blick eine Rücksicht auf Fremdes einschließt. Noch die Mißachtung enthält einen Funken von Achtung. Herstellung, die jede Response vermissen ließe, liefe hinaus auf eine bloße Reproduktion des Bestehenden, auf bloßes Überleben. Wenn das Leben mit einer besonderen Art von Pathos beginnt und endet, so käme jede Produktion des Lebens einer Selbstaufhebung des Lebens gleich. Sie wäre Ausdruck jenes Selbstwiderspruchs, den in Nietzsche in seiner Genealogie der Moral als "Leben gegen Leben" bezeichnet.

\section{Literatur}

DÖRNER, K., Der gute Arzt, Stuttgart/New York: Schattauer, 2001.

GEHRING, P., Was ist Biomacht? Vom zweifelhaften Mehrwert des Lebens, Frankfurt am Main/NewYork: Campus, 2006.

GEYER, Ch. (Hg.), Biopolitik, Frankfurt am Main: Suhrkamp, 2001.

HEIDEGGER, M., Die Frage nach der Technik, in: Vorträge und Aufsätze, Pfullingen: Neske, 1954.

HUSSERL, E. Ideen zu einer reinen Phänomenologie und phänomenologischen Philosophie, 2. Buch (Hua IV), Den Haag: M. Nijhoff, 1952.

LINDEMANN, G., Die Grenzen des Sozialen. Zur sozio-technischen Konstruktion von Leben und Tod in der Intensivmedizin, München: Fink, 2002.

MERLEAU-PONTY, M., Phénoménologie de la perception, Paris: Gallimard, 1945.

WALDENFELS, B., Der Stachel des Fremden, Frankfurt am Main: Suhrkamp, 1990.

- Topographie des Fremden, Frankfurt am Main: Suhrkamp, 1997.

. Grenzen der Normalisierung, Frankfurt am Main: Suhrkamp, 1998.

. Das leibliche Selbst, Frankfurt am Main: Suhrkamp, 2000.

. Bruchlinien der Erfahrung, Frankfurt am Main: Suhrkamp, 2002.

. Schattenrisse der Moral, Frankfurt am Main: Suhrkamp, 2006. 